\title{
Apendicitis aguda relacionado con endometriosis infiltrativa profunda: reporte de caso
}

\author{
Jesús A. Martín-Pérez ${ }^{*}$ Israel de Alba-Cruz, Jorge A. Domínguez-Rodríguez y Ángel J. Lara-Valdes ${ }^{1}$ \\ Departamento de Cirugía General, Hospital Regional General Ignacio Zaragoza, Instituto de Seguridad y Servicios Sociales de los Trabajadores \\ del Estado, Ciudad de México, México
}

\begin{abstract}
Resumen
La endometriosis es la presencia de glándulas endometriales y estroma fuera de la cavidad y musculatura uterina. La apendicitis aguda (AA) relacionada con la endometriosis infiltrativa profunda (EIP), descrita desde 1860 (por Rokitansy), se ha reportado en alrededor del 3\%. Objetivo: Presentar un caso de AA secundaria a EIP con clínica de abdomen agudo. Material y métodos: Paciente femenino de 41 años, que inicia con dolor abdominal compatible con $A A$, realizándose cirugía urgente. Resultados: El reporte histopatológico definitivo demuestra una EIP. Conclusión: Discutimos una variante rara de presentación AA secundaria a EIP. En mujeres en edad reproductiva son necesarios estudios complementarios e incluso exploración quirúrgica para la certeza diagnóstica.
\end{abstract}

Palabras clave: Endometriosis. Apendicitis aguda. Abdomen agudo. Hemicolectomía derecha.

\section{Acute appendicitis related to deep infiltrating endometriosis: A case report}

\section{Abstract}

Endometriosis is the presence of endometrial glands and stroma outside the uterine cavity and musculature. Acute appendicitis $(A A)$ related to deep infiltrative endometriosis (DIE); described since 1860, by Rokitansy, it has been reported in about $3 \%$. Objective: To present a case of AA secondary to DIE with symptoms of acute abdomen. Material and Methods: 41-yearold female, with abdominal pain compatible with $A A$, undergoing urgent surgery. Results: The definitive histopathological report reveals an exceptional cause of $A A$, concluding with DIE. Conclusions: We discuss a rare variant of $A A$ presentation secondary to DIE. In women of reproductive age, complementary studies and even surgical exploration are necessary for diagnostic certainty.

Key words: Endometriosis. Acute appendicitis. Acute abdomen. Right hemicolectomy.

\section{Introducción}

La endometriosis es la presencia de glándulas endometriales y estroma fuera de la cavidad y musculatura uterina ${ }^{1}$. A partir 1860, Rokitansy describe la endometriosis infiltrativa profunda (EIP), la cual define como la presencia de glándulas endometriales/estromales $\geq 5 \mathrm{~mm}$ más allá del tejido extrauterino/peritoneal ${ }^{2}$, con una prevalencia reportada entre $5-25 \%$. En

\section{Correspondencia:}

*Jesús A. Martín-Pérez

E-mail: chucho0892@gmail.com
Disponible en internet: 25-11-2021

Fecha de recepción: 21-06-2021

Fecha de aceptación: 17-08-2021 DOI: 10.24875/REIE.21000065
Rev Educ Investig Emer. 2021;3(4):222-225 www.medicinadeemergencias.com

2604-6520 @ 2021 Sociedad Mexicana de Medicina de Emergencias, AC. Publicado por Permanyer México SA de CV. Este es un artículo open access bajo la licencia CC BY-NC-ND (http://creativecommons.org/licenses/by-nc-nd/4.0/). 
1951, Collins, et al. reportaron un total de 150 casos en la literatura; cuatro años después, describió más de 50,000 evaluaciones patológicas aleatorias del apéndice e informó que la prevalencia de endometriosis apendicular era del $0.054 \% 3,4$. Sin embargo, existe reportes del 0 al $8 \%$ y hasta el $3 \%$ de las apendicitis aguda (AA) están en relación con la EIP ${ }^{5-8}$. La endometriosis apendicular no solo puede causar síntomas de AA, también dolor cíclico y crónico en el cuadrante inferior derecho, melena, hemorragia intestinal baja, invaginación intestinal cecal y perforación intestinal ${ }^{6,9}$. Por consecuencia, en la mayoría de los casos, requerirá de un manejo quirúrgico ${ }^{10,11}$. El objetivo del presente trabajo es presentar un caso de AA de causa inusual secundaria a patología ginecológica.

\section{Presentación del caso}

Se trata de una mujer con 41 años, sin antecedentes personales patológicos heredofamiliares o quirúrgicos de importancia para su padecimiento actual. Con talla $1.66 \mathrm{~cm}$ y peso $75 \mathrm{~kg}$ (índice de masa corporal 27.27). Antecedentes gineco-obstétricos: menarca a los 12 años, telarca 11 años, pubarca 12 años, patrón menstrual irregular e inicio de vida sexual a los 18 años, única pareja sexual. Método de planificación familiar: dispositivo intrauterino. Dos gestaciones, un parto y un aborto (espontáneo sin especificar). Al interrogatorio directo negó acudir a consultas de control ginecológico, citología exfoliativa o mamografía en el último año. Previas sin alteraciones.

Ingresó al servicio de urgencias con dolor abdominal súbito, tipo cólico de intensidad moderada en epigastrio que posteriormente se localiza hacia la fosa ilíaca derecha de 24 horas de evolución, acompañado de vómitos, náuseas, intolerancia oral y fiebre $\left(38.5^{\circ} \mathrm{C}\right)$. Exploración física: McBurney, Rovsing, Psoas, von Blumberg positivos, al tacto vaginal con flujo vaginal blanquecino, acuoso e inodoro; resto de exploración sin relevancia. Análisis de sangre con leucocitosis $(16,400 \mathrm{ul})$ y neutrofilia $(91 \%)$, con resto de resultados normales. La tomografía de abdomen reportó tumoración en fosa ilíaca derecha con dimensiones de $43 \times 31 \times 39 \mathrm{~mm}$ (Fig. 1), sugiriendo apendicitis aguda. Se realizó exploración abdominal abierta por probable apendicitis agu$\mathrm{da}$, encontrando masa tumoral absceda con abundante líquido libre, en fosa ilíaca derecha sin identificación clara de tejido cecal y apendicular dado adherencias interasas y asa-pared consecuente a tejido inflamatorio. De acuerdo con lo anterior, se decidió realizar hemicolectomía derecha (con una resección completa del

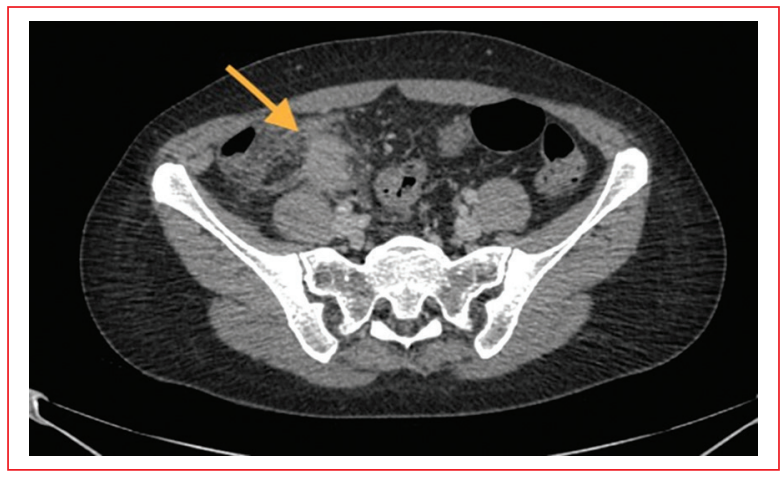

Figura 1. Tomografía computarizada simple de abdomen, corte axial en el cual se observa tumoración de $43 \times 31$ x $39 \mathrm{~mm}$ en fosa ilíaca derecha (flecha).

tumor sin linfadenectomía) con ileostomía tipo Brooke debido a cavidad abdominal contaminada secundario a peritonitis purulenta dado proceso infeccioso a nivel ileocecal, resto de exploración abdominal sin hallazgos relevantes. Posterior al lavado de cavidad abdominal y eliminación de placas de fibrina en región apendicular y anexo derecho. Se realizó una exploración pélvica, donde se observó: implantes de tejido con apariencia endometrial en peritoneo visceral del fondo de saco con predominio derecho y obliteración parcial, dos lesiones de aproximadamente $1.5 \mathrm{~cm}$ de diámetro heterogéneo en anexo derecho a nivel de ovario con adherencias firmes hacia la trompa ipsilateral, así como también posibles implantes en región del apéndice cecal y en recto. Se revisó resto de fondo de saco, útero y anexos izquierdos sin encontrar otras alteraciones estructurales de importancia. Se decidió realizar interconsulta transoperatoria al servicio de ginecología, sin embargo, no acuden por estar en procedimiento de urgencia, por lo que se decide finalizar el procedimiento quirúrgico y solicitar la valoración posquirúrgica.

\section{Resultados}

La paciente cursó con adecuada evolución postoperatoria, egresando al quinto día de estancia hospitalaria con subsecuente seguimiento por ginecología. El informe histopatológico definitivo reportó apendicitis reactiva secundaria a tejido endometrial, que compromete epiplón mayor, tejido periapendicular y pericecal (Figs. 2 y 3 ).

A los seis meses ingresó a nueva intervención quirúrgica para restitución del tránsito intestinal mediante una ileotransverso anastomosis termino-lateral sin complicaciones. Permaneció en seguimiento por el servicio 


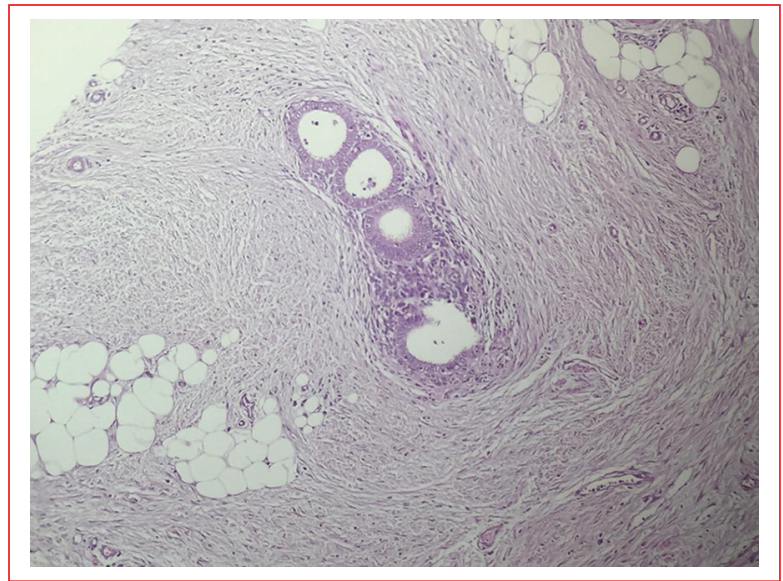

Figura 2. Los cortes histológicos con hematoxilina y eosina muestran en todo el espesor de la pared del apéndice algunas islas compuestas por glándulas tubulares revestidas por una capa de células cilíndricas con moderado citoplasma eosinófilo, los núcleos se encuentran dispuestos de manera pseudoestratificada $\sin$ atipia.

de ginecología con tratamiento anticonceptivo hormonal oral y con remisión de cuadros de dolor pélvico a los seis meses de seguimiento.

\section{Discusión}

La endometriosis se caracteriza por un tejido similar al endometrio localizado fuera del útero; principalmente en el peritoneo pélvico, ovarios, tabique rectovaginal, y en casos raros en el diafragma, pleura y pericardio. Se presenta en el 50 al $60 \%$ de las mujeres y adolescentes con dolor pélvico crónico, y del 6 al $15 \%$ de las mujeres en edad reproductiva ${ }^{1,6}$. Afecta al intestino en el $3-37 \%$ de todos los casos de estos; el $90 \%$ con involucro al recto o el colon sigmoides ${ }^{12}$.

Sobre la patogénesis existen tres teorías principales: menstruación retrógrada con implantación y falla del aclaramiento inmunitario, metaplasia celómica y la metástasis hematológica o linfática ${ }^{1}$. Explicando que los síntomas son por una reacción esclerosante en la pared que conduce a estrechez intraluminal. Por lo tanto, en eventos repetitivos genera una inflamación crónica periapendicular desarrollando incluso tumores que requieren manejo quirúrgico radical ${ }^{10}$. La endometriosis del apéndice es extremadamente rara, con una prevalencia de alrededor del $2.8 \%$ de las mujeres con endometriosis y del $0.4 \%$ de las mujeres en la población general ${ }^{5-8}$. En dos análisis retrospectivos realizados por Dincel, et al. y Unver, et al. se estudiaron 1,970 y

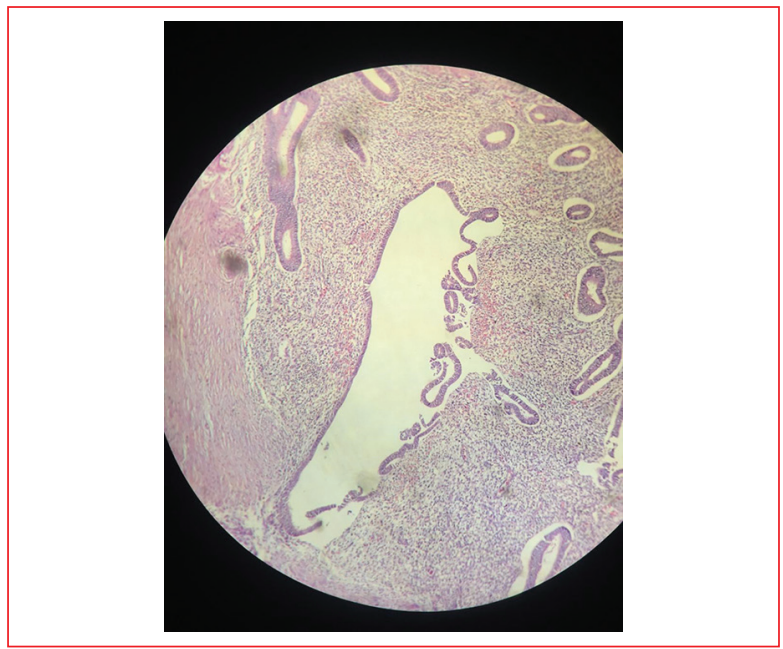

Figura 3. Dichas glándulas están inmersas en un estroma de tipo endometrial en donde se observan algunos macrófagos con pigmento de hemosiderina en su citoplasma y algunos eritrocitos extravasados.

2,047 pacientes respectivamente con reporte histopatológico de apendicitis aguda por diferentes causas, encontrando solamente un caso en cada estudio (1.7 y $0.05 \%$ respectivamente) secundario a EIP ${ }^{13,14}$. Lo mencionado anteriormente apoya el hecho de que sigue siendo una patología infrecuente y que continúa en temas de investigación hasta la actualidad.

Por lo general, los síntomas de esta entidad son: dolor abdominal de repetición o cíclico, fiebre, náuseas y recuento elevado de glóbulos blancos; sin embargo, es necesario realizar estudios de gabinete para confirmar el diagnóstico y planear una intervención quirúrgica adecuada ${ }^{15}$. Un estudio que incluyó a 195 pacientes con sospecha de endometriosis demostró que la resonancia magnética (RM) tiene una sensibilidad del $88 \%$ y una especificidad del $98 \%$ en el diagnóstico de la endometriosis intestinal, ratificando a la RM como uno de los estudios con mayor confiabilidad $2,16,17$; sin embargo, el diagnóstico certero requiere un examen histopatológico definitivo ${ }^{13,18}$.

La clasificación de la American Fertility Society (AFS), la cual se ha utilizado convencionalmente para estadificar la endometriosis, se divide en cuatro estadios (estadio I/mínimo, estadio II/leve, estadio III/moderado y estadio IV/severo). Sin embargo, focos de endometriosis fuera del aparato reproductor de la mujer, como los que involucran la cavidad intraperitoneal, el intestino, la vejiga o el uréter y la adenomiosis extensa, no están adecuadamente representados por la puntuación AFS ${ }^{19}$. Por lo tanto, se sugiere un sistema de clasificación actualizado 
basado en cambios anatómicos para evaluar de manera más efectiva las estructuras patológicas. En relación con esto, actualmente se discute la necesidad de una clasificación alternativa 0 adicional de EIP20,21.

Por último, el tratamiento de la AA secundario a EIP es la apendicectomía, ileocectomía o hemicolectomía derecha de acuerdo con los hallazgos encontrados, con una exploración de la cavidad abdominal evaluando extensión de la enfermedad. Hasta el momento la tasa de recurrencia se ha informado en el $4.7-25 \%$ en un seguimiento mayor a dos años $22-24$.

\section{Conclusión}

El diagnóstico diferencial de abdomen agudo es amplio, es por ello que ante la incertidumbre diagnóstica y como parte del protocolo y manejo de AA recomendamos una exploración pélvica abdominal completa, ya sea de abordaje abierto o laparoscópico en mujeres jóvenes en edad reproductiva y con alta sospecha de EIP.

\section{Agradecimientos}

A los servicios de anestesiología, imagenología, patología y ginecología, por su siempre incondicional apoyo.

\section{Financiamiento}

Los autores no recibieron financiamiento para llevar a cabo este artículo.

\section{Conflicto de intereses}

Los autores declaran no tener conflicto de intereses alguno.

\section{Responsabilidades éticas}

Protección de personas y animales. Los autores declaran que para esta investigación no se han realizado experimentos en seres humanos ni en animales.

Confidencialidad de los datos. Los autores declaran que han seguido los protocolos de su centro de trabajo sobre la publicación de datos de pacientes.

Derecho a la privacidad y consentimiento informado. Los autores han obtenido el consentimiento informado de los pacientes y/o sujetos referidos en el artículo. Este documento obra en poder del autor de correspondencia.

\section{Bibliografía}

1. Giudice LC. Endometriosis: Clinical practice. $N$ Engl J Med. 2010;362(25):2389-99.

2. Darvishzadeh A, McEachern W, Lee TK, Bhosale P, Shirkhoda A, Menias C, et al. Deep pelvic endometriosis: a radiologist's guide to key imaging features with clinical and histopathologic review. Abdom Radiol. 2016;41(12):2380-400.

3. Lane RE. Endometriosis of the vermiform appendix. Am J Obstet Gynecol. 1960;79(2):372-7.

4. Collins DC. A study of 50,000 specimens of the human vermiform appendix. Surg Gynecol Obstet. 1955;101(4):437-45.

5. St. John BP, Snider AE, Kellermier H, Minhas S, Nottingham JM. Endometriosis of the appendix presenting as acute appendicitis with unusual appearance. Int J Surg Case Rep. 2018;53:211-3.

6. Dickson-Lowe RA, Ibrahim S, Munthali L, Hasan F. Intussusception of the vermiform appendix. BMJ Case Rep. 2015;2015:bcr2014207584.

7. Emre A, Akbulut S, Yilmaz M, Bozdag Z. An unusual cause of acute appendicitis: Appendiceal endometriosis. Int J Surg Case Rep. 2013;4(1):54-7.

8. Gustofson RL, Kim N, Liu S, Stratton P. Endometriosis and the appendix: a case series and comprehensive review of the literature. Fertil Steril. 2006;86(2):298-303.

9. Berker B, LaShay N, Davarpanah R, Marziali M, Nezhat CH, Nezhat C. Laparoscopic appendectomy in patients with endometriosis. J Minim Invasive Gynecol. 2005;12(3):206-9.

10. Laskou S, Papavramidis TS, Cheva A, Michalopoulos N, Koulouris C Kesisoglou I, et al. Acute appendicitis caused by endometriosis: A case report. J Med Case Rep. 2011;5:1-5.

11. Villarreal-Peral C, Olvera-Gracida L, de Lourdes González-Maynes M, Saucedo-Ruiz G. Endometriosis apendicular como causa de abdomen agudo. Ginecol Obstet Mex. 2011;79(8):489-92.

12. Guven H, Koc B, Saglam F, Bayram IA, Adas G. Emergency right hemicolectomy for inflammatory cecal masses mimicking acute appendicitis. World J Emerg Surg. 2014;9(1):1-5.

13. Alizadeh Otaghvar $H$, Hosseini M, Shabestanipour G, Tizmaghz A, Sedehi Esfahani G. Cecal endometriosis presenting as acute appendicitis. Case Rep Surg. 2014;2014:1-3.

14. Revised American Society for Reproductive Medicine classification of endometriosis: 1996. Fertil Steril. 1997;67(5):817-21.

15. Keckstein J, Becker CM, Canis M, Feki A, Grimbizis GF, Hummelshoj L, et al. Recommendations for the surgical treatment of endometriosis. Part 2: deep endometriosis. Hum Reprod Open. 2020;2020(1):1-25.

16. Bouquet de Joliniere J, Major A, Ayoubi JM, Cabry R, Khomsi F, Lesec G, et al. It is necessary to purpose an add-on to the american classification of endometriosis? This disease can be compared to a malignant proliferation while remaining benign in most cases. EndoGram ${ }^{\circledR}$ is a new profile witness of its evolutionary potential. Front Surg. 2019;6:27.

17. Dincel O, Goksu M, Turk BA, Pehlivano囚lu B, I囚ler S. Unexpected findings in the routine histopathological examinations of appendectomy specimens A retrospective analysis of 1,970 patients. Ann Ital Chir. 2017;88(6):519-25.

18. Unver N, Coban G, Arıcı DS, Buyukpınarbasılı N, Gucin Z, Malya FÜ, et al. Unusual histopathological findings in appendectomy specimens: A retrospective analysis of 2047 cases. Int J Surg Pathol. 2019;27(2):142-6.

19. Shen AY, Stanes A. Isolated appendiceal endometriosis. J Obstet Gynaecol Can. 2016;38(10):979-81.

20. Bazot M, Darai E, Hourani R, Thomassin I, Cortez A, Uzan S, et al. Deep pelvic endometriosis: MR imaging for diagnosis and prediction of extension of disease. Radiology. 2004;232(2):379-89.

21. Hines JJ, Paek GK, Lee P, Wu L, Katz DS. Beyond appendicitis; radiologic review of unusual and rare pathology of the appendix. Abdom Radiol. 2016;41(3):568-81.

22. Campagnacci R, Perretta S, Guerrieri M, Paganini AM, De Sanctis A, Ciavattini A, et al. Laparoscopic colorectal resection for endometriosis. Surg Endosc Other Interv Tech. 2005;19(5):662-4.

23. Wolthuis AM, Meuleman C, Tomassetti C, D'Hooghe T, de Buck van Overstraeten A, D'Hoore A. Bowel endometriosis: Colorectal surgeon's perspective in a multidisciplinary surgical team. World $\mathrm{J}$ Gastroenterol. 2014;20(42):15616-23.

24. Saleem A, Navarro P, Munson JL, Hall J. Endometriosis of the appendix: Report of three cases. Int J Surg Case Rep. 2011;2(2):16-9. 